\title{
DIRECTLY RECORDING DIFFRACTION PHENOMENA IN THE TIME DOMAIN
}

\author{
M. Lõhmus ${ }^{\mathrm{a}}$, P. Bowlan ${ }^{\natural}$, R. Trebino ${ }^{\natural}$, H. Valtna-Lukner ${ }^{\mathrm{a}}$, P. Piksarv ${ }^{\mathrm{a}}$, and P. Saari ${ }^{\mathrm{a}}$

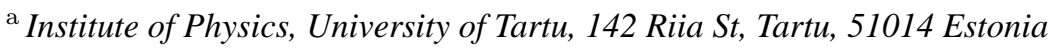 \\ E-mail:madisl@ut.ee \\ ${ }^{\mathrm{b}}$ School of Physics, Georgia Institute of Technology, 837 State St NW, Atlanta, GA 30332, USA
}

Received 15 October 2009; accepted 19 March 2010

\begin{abstract}
The wave-field produced by a $\sim 30$ fs duration Ti:sapphire oscillator pulse behind a circular aperture and circular opaque disk is measured using the ultrashort-laser-pulse measurement technique, scanning SEA TADPOLE. The high spatial and temporal resolution of the measuring technique enables us to fully image the diffracted field behind the apertures and record the interference pattern produced by the so-called boundary diffraction wave pulses.
\end{abstract}

Keywords: diffraction, interference

PACS: $42.25 . \mathrm{Fx}, 42.25 . \mathrm{Gy}, 42.25 . \mathrm{Hz}$

\section{Introduction}

Throughout the history of describing and viewing the effects of diffraction, it has usually been done by using monochromatic light in order to make the diffraction pattern visible. However, using ultrashort laser pulses and advanced techniques for measurement, it is now possible to study diffraction in the time domain. The diffraction of femtosecond pulses was theoretically studied in [1] in the context of boundary diffraction wave theory, where it was shown that, for the special case of a circular aperture, with incident fields having axial symmetry around the optical axis, the so-called geometrical wave (geometric (direct) pulse) $U_{\mathrm{G}}$ and the boundary wave (boundary wave pulse) $U_{\mathrm{B}}$ could have comparable amplitudes on the optical axis. Three cases for the incident femtosecond pulse front - plane, divergent, and convergent pulse fronts - were studied and it was shown that, when the pulses are short enough, one could have two pulses with the same temporal profile and comparable intensities propagating along the optical axis after the diffractive element. One pulse is caused by the incident field that goes through the aperture undisturbed and the other is caused by the socalled boundary diffraction wave pulse. The diffracted field is said to arise from the superposition of the direct and the boundary diffraction wave pulse. A later study [2] reported the experimental demonstration of the existence of the boundary wave pulse by measuring the modulated spectrum on the optical axis (caused by the two separate pulses) and the integrated radial intensity distribution of the diffracted field behind a circular aperture.

In this paper we present the results of the direct measurement of the diffracted field behind a circular aperture and circular opaque disk using high spectral (temporal) and spatial resolution provided by the ultrashort pulse measurement method called scanning SEA TADPOLE [3]. The short nature of the pulses used in the experiment, combined with the high spatiotemporal resolution and sensitivity of the method used for measurement, enables us to fully image the boundary wave pulse and the direct pulse separated in time behind the circular aperture and opaque circular disk. Taking measurements at different distances from the apertures enables us to study the temporal evolution of the diffraction of the propagating ultrashort laser pulses, and show the superluminal speed of the boundary wave pulse on the optical axis.

\section{Numerical simulations}

We performed numerical simulations for the diffracted field behind the apertures on the basis of the equation (12) in [1] derived from the work of Miyamoto and Wolf [4]. According to the boundary diffraction wave theory, the field of the boundary 


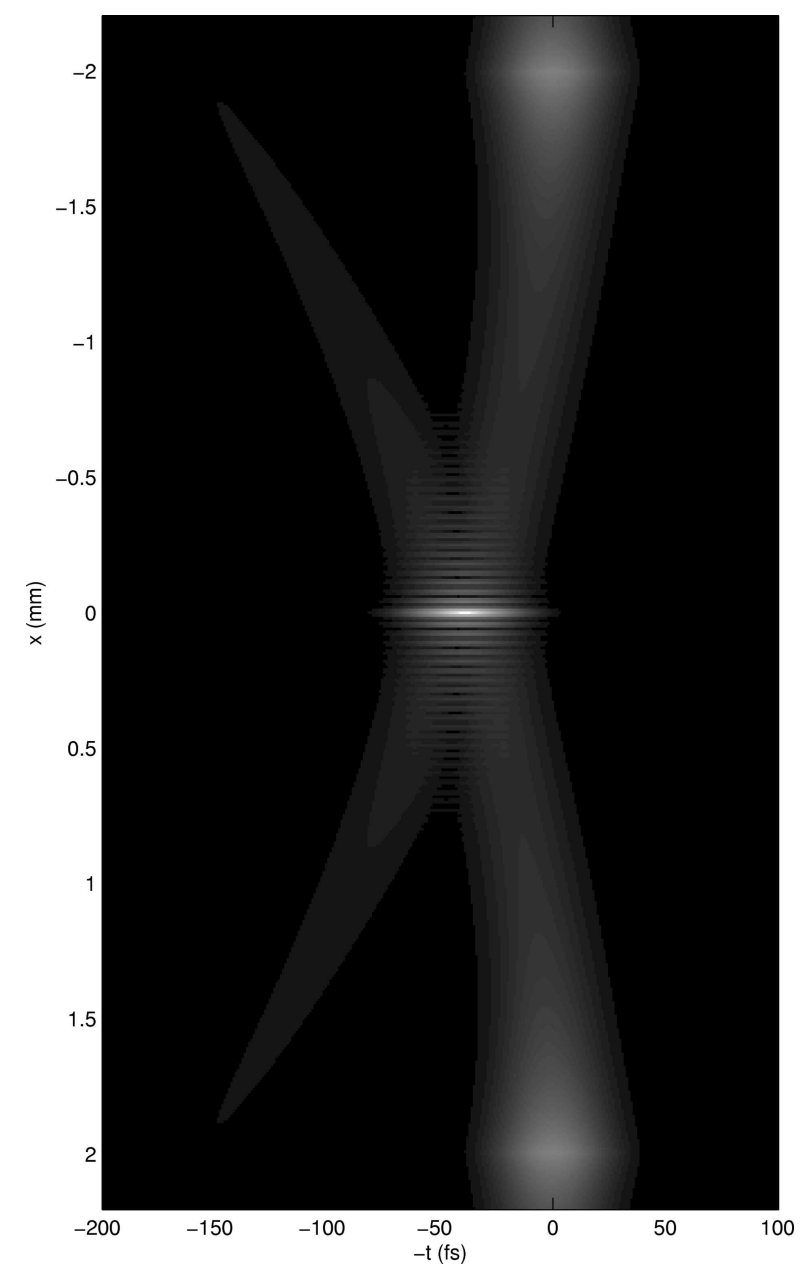

Fig. 1. The numerical simulation of a boundary diffraction wave pulse at $17 \mathrm{~cm}$ behind a $4 \mathrm{~mm}$ circular aperture under pulsed illumination. The interference fringes in the middle are caused by the interference of the elementary boundary diffraction waves originating from the edges of the aperture.

diffraction wave pulse behind a circular aperture with incident fields having axial symmetry around the optical axis can be expressed as

$$
\begin{aligned}
& u_{\mathrm{B}}(r, z, t)=\frac{u_{0} \mathrm{e}^{\mathrm{i} \omega_{0} t}}{2 \pi} \\
& \times \int_{0}^{\pi} v\left(t-\frac{s(\psi)}{c}\right) \mathrm{e}^{-\mathrm{i} k_{0} s(\psi)}\left(1+\frac{z}{s(\psi)}\right) g(K, \psi) \mathrm{d} \psi,
\end{aligned}
$$

where $v(t)$ is the temporal envelope of the incident pulse, $\omega_{0}$ is the central angular frequency, and $k_{0}$ is the wave number at $\omega_{0}$. The expression $s(\psi)=$ $\sqrt{z^{2}+a^{2}+r^{2}-2 a r \cos (\psi)}, K=r / a$ is a dimensionless variable, and $g(K, \psi)=[K \cos (\psi)-1] /[1+$ $\left.K^{2}-2 K \cos \psi\right]^{-1}$. The structure of the boundary-wave pulse in the case of a plane wave illumination of a circular aperture can be seen on Fig. 1. The cylindrical symmetry of the aperture leads to constructive interference of the elementary boundary diffraction waves originating from the edge of the aperture on the axis of symmetry of the circular aperture. This causes the boundary diffraction wave to have significant intensity on the optical axis that is comparable to the intensity of the direct pulse. The diffracted field is then calculated as the sum of the fields of the boundary wave pulse and direct pulse:

$$
u(P, t)=u_{\mathrm{G}}(P, t)+u_{\mathrm{B}}(P, t),
$$

where $P \equiv(x, y, z)$ is the point of measurement behind the aperture and $u_{\mathrm{G}}$ is calculated as

$$
u_{\mathrm{G}}=\left\{\begin{array}{cl}
u_{i}(P, t), & \text { if } P \text { is in the direct beam }, \\
0, & \text { if } P \text { is in the shadow, }
\end{array}\right.
$$

where $u_{i}(P, t)=u_{0} h(t-z / c)$ is the incident pulse. It is evident that the direct pulse in this theory has a discontinuity on the edge of the aperture. This is compensated by the discontinuity of the boundary wave pulse across the aperture (in the centre of the brighter areas of the boundary diffraction wave near the edge of the aperture on Fig. 1) so the resulting diffracted field is a continuous function of the position.

This equation for nonmonochromatic fields for circular apertures assumes that the incident field is a plane wave (pulse) with a normal incidence. In the case of laser-produced Gaussian beams, this assumption does not exactly hold, but for truncated Gaussian beams the boundary diffraction still appears as shown in [5]. A comparison of measurements and simulations shows that the error produced when the pulse fronts differ slightly from a plane wave is negligible.

\section{Scanning SEA TADPOLE}

In order to record the complex structure of the diffraction pattern behind the apertures, high spatial and temporal (spectral) resolutions are needed. An experimentally simple version of spectral interferometry called SEA TADPOLE was the first technique shown to be able to directly measure the spatiotemporal electric field of focusing ultrashort pulses [6]. The small mode size of the optical fibres used in this method naturally enables electric fields of ultrashort pulses to be measured with high spatial resolution. In a SEA TADPOLE set-up, the previously characterized reference pulse and the unknown pulse are coupled into two identical fibres as seen in Fig. 2. The entrance of the unknown 


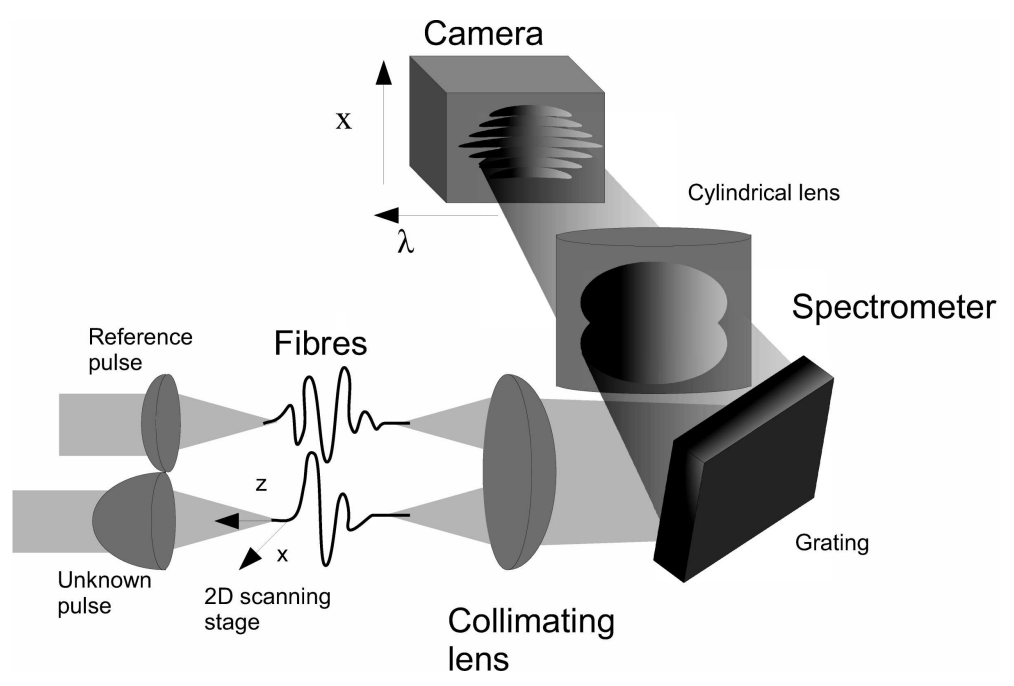

Fig. 2. Scanning SEA TADPOLE set-up. A reference pulse and an unknown pulse are coupled into two single-mode optical fibres. The diverging beams from the fibres are collimated with a spherical lens. The collimated beams cross and the interference pattern is recorded with a camera. In horizontal plane a grating and a cylindrical lens are used to make a spectrometer and to map the wavelength onto the camera's horizontal axis.

pulse's fibre is placed on a scanning stage. The reference pulse's fibre entrance and both of the fibres ends are fixed. The diverging beams from the fibres are collimated vertically by a spherical lens, and the collimated beams cross and interfere. The interference pattern is recorded with a camera. In the horizontal plane, a grating and a cylindrical lens are used to make a spectrometer and to map wavelength on the camera's horizontal axis. This interferogram, recorded for every position of the scanning stage, allows one to reconstruct the full spatiotemporal field $E(x, y, z, t)$.

\section{Measurements}

The experiments were carried out using KM Labs Ti:sapphire oscillator with $\sim 37 \mathrm{~nm}$ FWHM bandwidth spectrum with a central wavelength of $805 \mathrm{~nm}$ and pulse duration of about $30 \mathrm{fs}$. The temporal resolution of our SEA TADPOLE set-up was 4.6 fs. The circular aperture and opaque disk were placed in the unknown pulse arm of the SEA TADPOLE set-up, where the optical fibre was placed on the 2D scanning stage. The unmodified pulse (pulse directly from the laser) was sent to the second fibre and was used as a reference. For each aperture, the tip of the scanning fibre was first moved into the bright central spot on the optical axis caused by the interference of the elementary boundary wave pulses. The fibre was then moved horizontally back over the edge of the aperture, and a full scan on the axis perpendicular to the pulse propagation was made to produce the images. Due to the cylindrical symmetry of the apertures and the resulting diffracted field, only one scan in the plane of the aperture diameter (that is, for various longitudinal distances after the aperture and various transverse distances parallel to the plane of the aperture) was necessary to obtain the full information of the field. Measurements were taken at different distances from the aperture and disk to study the time evolution of the diffraction of the ultrashort pulses.

The spatial resolution of a SEA TADPOLE set-up is determined by the mode size of the scanning fibre [6]. In our set-up we used a fibre that had a core diameter of $5.4 \mu \mathrm{m}$, which is also the achieved spatial resolution of our measurements. Our step size of each consecutive measurement in one scan was smaller than the actual spatial resolution to obtain smoother images of the interference pattern of the diffracted field.

\section{Discussion}

For the circular opaque disk, measurements at distances of 92, 172, and $252 \mathrm{~mm}$ are shown in Fig. 3 along with the simulations. The circular aperture measurements form distances of 25,55 , and $85 \mathrm{~mm}$ are shown in Fig. 4. The intensities in every image are in arbitrary units with maximum of 1 . The scanning range of the fibre for the circular opaque disk was $8 \mathrm{~mm}$ and for the circular aperture $4.4 \mathrm{~mm}$. Each image displays the amplitude of the electric field (square root of the intensity) versus $-t$ so that the parts of the diffracted field that arrive earlier in time appear on the right-hand side of the plots.

In the pictures it is easy to see two diffraction patterns. One has a lower spatial modulation on the edges 

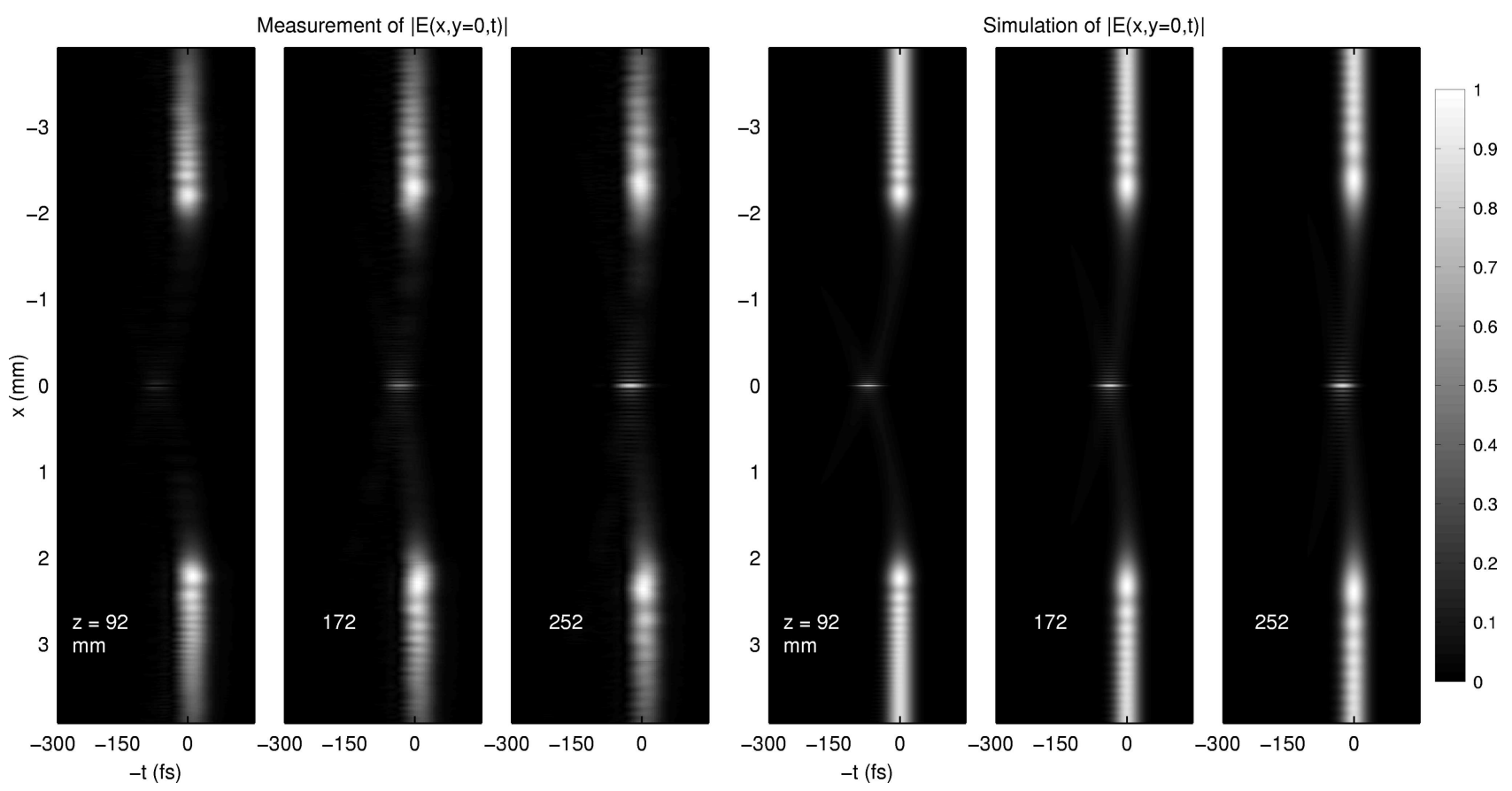

Fig. 3. The measured (left) and calculated (right) fields behind a $4 \mathrm{~mm}$ circular disk at different distances.
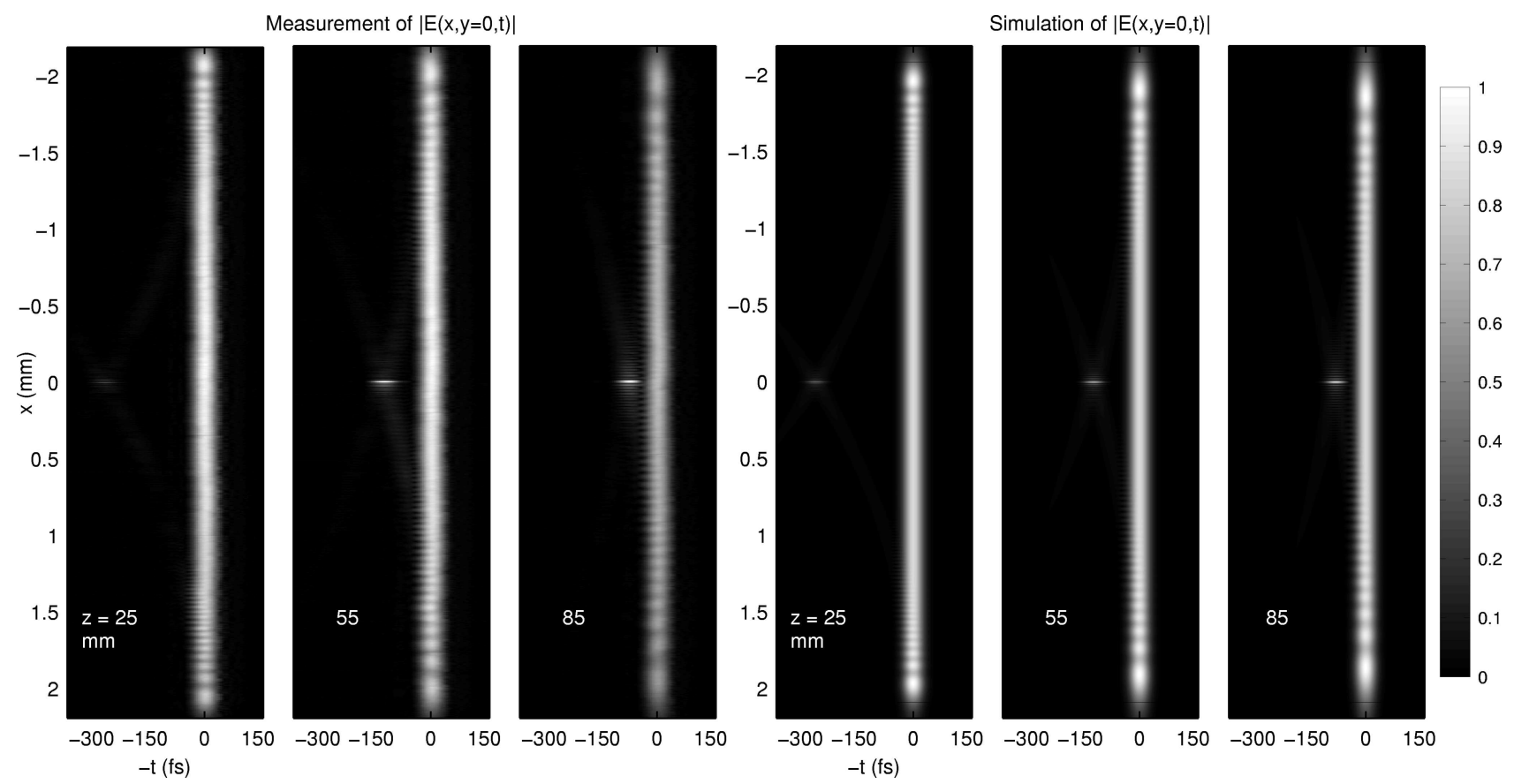

Fig. 4. The measured (left) and calculated (right) fields behind a $4 \mathrm{~mm}$ circular aperture at different distances.

of the direct pulse that is caused by the interference between boundary wave pulse and the direct pulse. Interference fringes can also be seen around the bright central spot analogous to the Bessel-X pulses [7, 8], which are caused by the interference between the elementary boundary diffraction waves originating from the edge of the aperture. Also similar to the Bessel-
$\mathrm{X}$ pulse the boundary wave pulse has a letter X-like axial cross-section and propagates on the optical axis superluminally. But while the group velocity of the former has a constant value exceeding $c$, the central spot of the boundary wave pulse is decelerating (see also [1, 9,-11]). The elementary boundary diffraction waves emanating from the edges of the aperture must 
travel a longer distance to reach the optical axis compared to the centre of the direct pulse and thus lag behind. The difference in distance they need to travel compared to the distance travelled by the direct pulse decreases as the pulse propagates. The superluminal speed can be easily seen as the constructive interference of the boundary wave pulse on the optical axis catching up with the direct pulse that has the propagation speed of $c$.

While the theoretical study shows that, when the laser pulses are short enough to separate the boundary wave pulse from the direct pulse, the two pulses propagating on the optical axis should have comparable intensities, this is not the case in our experiments. This is due to the fact that the transverse intensity profile of the pulses was Gaussian, with FWHM about $4 \mathrm{~mm}$. So the pulse intensity on the aperture was approximately one half of that at the centre of the pulse. As a result, in our measurements, the intensity of the boundary wave pulse or the constructive interference of the elementary boundary-wave pulse on the optical axis is smaller than the intensity of the direct pulse on the optical axis. Also the accurate measurement of the intensity of the boundary wave pulse is difficult to accomplish since, at the maximum distance from the aperture (about $25 \mathrm{~cm}$ ) where the interference pattern has the lowest spatial modulation, the size of the central bright spot of the interference pattern is still only about $50 \mu \mathrm{m}$. So any deviation from exact alignment of the scanning fibre tip from the plane of the diameter of the aperture will inevitably cause the maximum intensity of the spot to be inaccurately measured. This becomes even more critical when one considers measurements that are done closer to the aperture where the fringe spacing of the diffraction pattern becomes denser and fringes themselves smaller.

\section{Conclusion}

The complete diffracted field, $E(x, y, z, t)$, of ultrashort laser pulses behind circular opaque disk and circular aperture is measured with high spectral and temporal resolution using a method called scanning SEA TADPOLE. The pulsed illumination of the apertures allows us to image the so-called boundary diffraction wave separately from the direct pulse. The high spatial resolution reveals the fine diffraction fringes caused by the interference of the elementary boundary wave pulses near the optical axis. We also carried out numerical simulations based on the boundary diffraction

wave theory. The simulations and the experimental data are found to be in good agreement.

\section{References}

[1] Z.L. Horváth and Zs. Bor, Diffraction of short pulses with boundary diffraction wave theory, Phys. Rev. E 63(2), 026601-1-11 (2001).

[2] Z.L. Horváth, J. Klebniczki, G. Kurdi, and A.P. Kovács, Experimental investigation of the boundary wave pulse, Opt. Commun. 239(4-6), 243-250 (2004).

[3] P. Bowlan, P. Gabolde, M.A. Coughlan, R. Trebino, and R.J. Levis, Measuring the spatiotemporal electric field of ultrashort pulses with high spatial and spectral resolution, J. Opt. Soc. Am. B 25(6), A81-A91 (2008).

[4] K. Miyamoto and E. Wolf, Generalization of the Maggi-Rubinowicz theory of the boundary diffraction wave - Part II, J. Opt. Soc. Am. 52(6), 626-637 (1962).

[5] Z.L. Horváth and Zs. Bor, Reshaping of femtosecond pulses by the Gouy phase shift, Phys. Rev. E 60(2), 2337-2346 (1999).

[6] P. Bowlan, P. Gabolde, and R. Trebino, Directly measuring the spatio-temporal electric field of focusing ultrashort pulses, Opt. Express 15(16), 10219-10230 (2007).

[7] P. Saari and K. Reivelt, Evidence of X-shaped propagation-invariant localized light waves, Phys. Rev. Lett. 79(21), 4135-4138 (1997).

[8] P. Bowlan, H. Valtna-Lukner, M. Lõhmus, P. Piksarv, P. Saari, and R. Trebino, Measuring the spatiotemporal field of ultrashort Bessel-X pulses, Opt. Lett. 34(15), 2276-2278 (2009).

[9] P. Saari and K. Reivelt, Generation and classification of localized waves by Lorentz transformations in Fourier space, Phys. Rev. E 69(3), 036612-1-12 (2004).

[10] M. Clerici, D. Faccio, A. Lotti, E. Rubino, O. Jedrkiewicz, J. Biegert, and P. Di Trapani, Finite-energy, accelerating Bessel pulses, Opt. Express 16(24), 19807-19811 (2008).

[11] H. Valtna-Lukner, P. Bowlan, M. Lõhmus, P. Piksarv, R. Trebino, and P. Saari, Direct spatiotemporal measurements of accelerating ultrashort Bessel-type light bullets, Opt. Express 17(17), 14948-14955 (2009). 


\title{
TIESIOGINIS DIFRAKCIJOS REIŠKINIŲ REGISTRAVIMAS LAIKINĖJE SRITYJE
}

\author{
M. Lõhmus ${ }^{\text {a }}$, P. Bowlan ${ }^{\text {b }}$, R. Trebino ${ }^{\text {b }}$, H. Valtna-Lukner ${ }^{\text {a }}$, P. Piksarv ${ }^{\text {a }}$, P. Saari ${ }^{\text {a }}$ \\ ${ }^{\text {a }}$ Tartu universiteto fizikos institutas, Tartu, Estija \\ b Džordžijos technologijos universiteto Fizikos mokykla, Atlanta, JAV
}

\section{Santrauka}

Bangų laukas, sukurtas $\sim 30$ fs trukmès Ti:safyro osciliatoriaus impulsais už apskritos apertūros ir apskrito neperšviečiamo disko, matuojamas ultratrumpuju lazerio impulsų metodu, kuris angliškai vadinamas skenuojančiuoju SEA TADPOLE. Taikyto metodo didelè erdvinè ir laikiné skyra leidžia atvaizduoti visą už apertūrų difragavusị lauką ir užfiksuoti interferencinị vaizdą, susidarantị dèl vadinamųjų kraštinių difragavusių bangų impulsų. 